\section{Stoßwellentherapie: Labsal für schmerzende Füße}

\begin{abstract}
Fokussiert, energiereich und ohne Anästhesie - so kann die extrakorporale Stoßwellentherapie (ESWL) eine therapierefraktäre Plantarfasziitis deutlich lindern.
\end{abstract}

$\mathrm{D}$ ie meisten Patienten mit Plantarfasziitis sprechen zwar auf eine Behandlung mit Dehnübungen, NSAR oder Kortikoidinjektionen gut an, bei rund $10 \%$ versagen solche Therapien jedoch. Oft bleibt ihnen dann nur noch der Weg zum Chirurgen. Die Evidenz zur extrakorporale Stoßwellentherapie (ESWL) in dieser Indikation eher gering.

In einer placebokontrollierten Studie hat das Team um Dr. Hans Gollwitzer vom Klinikum Rechts der Isar in München geprüft, ob die ESWL bei therapierefraktären Beschwerden der Plantarsehne wirksam ist. Die Behandlung erfolgte mit 2.000 Impulsen (vier Impulse pro Sekunde; $0,25 \mathrm{~mJ} / \mathrm{mm}^{2}$ ) an der schmerzempfindlichsten Stelle und ohne Lokalanästhesie. Teilnehmer waren 250 Patienten mit chronischer Plantarfasziitis, die über mindestens sechs Monate hinweg auf vier oder mehr konventionelle Therapieversuche nicht angesprochen hatten. Die Hälfte der Patienten bekam drei Stoßwellenbehandlungen im Abstand von je einer Woche, die übrigen Teilnehmer eine Scheinbehandlung. Dabei wurde ein luftgefüllter Abstandhalter verwendet, der eine Übertragung der Stoßwellen verhinderte. Von außen sahen alle verwendeten Geräte gleich aus, sodass die Patienten nicht erkennen konnten, ob sie eine Stoßwellentherapie bekamen oder nicht.

Zwölf Wochen nach der letzten Behandlung erfolgte die Auswertung. Im Median gingen die Fersenschmerzen mit der Stoßwellentherapie gemessen anhand einer visuellen Analogskala um 69\% zurück, mit der Scheinbehandlung 35\%. Die Durchschnittswerte reduzierten sich jeweils um 55 und $40 \%$, die Unterschiede zwischen den beiden Verfahren waren signifikant ( $\mathrm{p}=0,0027)$.

Auch die funktionelle Verbesserung gemäß dem Roles-and-Maudsley-Score fiel in der Gruppe mit Stoßwellentherapie besser aus: Der Wert fiel von 3,6 auf
2,5 Punkte, in der Kontrollgruppe ging er von 3,7 auf 2,9 Punkte zurück. Der Unterschied bei der funktionellen Verbesserung von 0,4 Punkten war ebenfalls signifikant $(p=0,0006)$.

Deutlich mehr Patienten in der Gruppe mit Stoßwellentherapie (54 versus 37\%) sprachen auf das Verfahren mit einer mindestens $60 \%$ Schmerzreduktion an. Ferner hatten Patienten mit Stoßwellenbehandlung signifikant häufiger eine gute oder sehr gute Wirksamkeit verglichen mit einer Scheinbehandlung (74 versus $54 \%$ ).

48\% der Patienten mit Stoßwellentherapie, aber nur 33\% mit Scheinbehandlung waren mit dem Ergebnis zufrieden oder sehr zufrieden. $89 \%$ unter ESWL und $91 \%$ mit Placebo sprachen von einer guten Verträglichkeit der Methode.

Patienten beider Gruppen, die nach zwölf Wochen auf die Behandlung angesprochen hatten, waren bei der Nachuntersuchung nach einem Jahr praktisch beschwerdefrei.

Thomas Müller

Gollwitzer H et al. Clinically Relevant Effectiveness of Focused Extracorporeal Shock Wave Therapy in the Treatment of Chronic Plantar Fasciitis. J Bone Joint Surg Am 2015; 97(9): 701-708.

\section{Jogging light für den höchsten Lebenszeitgewinn}

Am längsten leben die Jogger, die ihren Lauf bedächtig angehen, bestätigt die Auswertung einer prospektiven Beobachtungsstudie zur Kausalität zwischen Jogging und Mortalität. Optimal ist danach eine Dauer von insgesamt 1 bis 2,4 Stunden an maximal drei Tagen pro Woche in langsamem bis durchschnittlichem Tempo.

$\mathrm{H}$ ierzulande schnüren dem Deutschen Leichtathletik-Verband zufolge etwa 10\% der Bevölkerung ab 14 Jahren regelmäßig die Laufschuhe. Das hat Folgen für die Gesundheit. Die Copenhagen City Heart Study hat gezeigt, dass joggende Männer ihr Leben um 6,2 Jahre und Frauen um 5,6 Jahre verlängern können. Die besten Chancen hatten der Studie zufolge Läufer, die maximal dreimal pro Woche in langsamem Tempo für bis zu zweieinhalb Stunden ihre Kreise zogen. Mehr davon ließ die Vor- teile dagegen hinter den Nachteilen verblassen. Peter Schnohr vom Frederiksberg Hospital in Kopenhagen und Kollegen deckten diesen U-förmigen Zusammenhang an insgesamt 1.098 gesunden Joggern zwischen 20 und 86 Jahren und 413 bewegungsarmen Nichtjoggern (Durchschnittsalter 61). Vom Zeitpunkt der ersten Untersuchung zwischen 2001 und 2003 beobachteten sie die Probanden bis maximal April 2013. Dabei wurden die Jogger in drei Leistungsgruppen eingeteilt: Jogging light

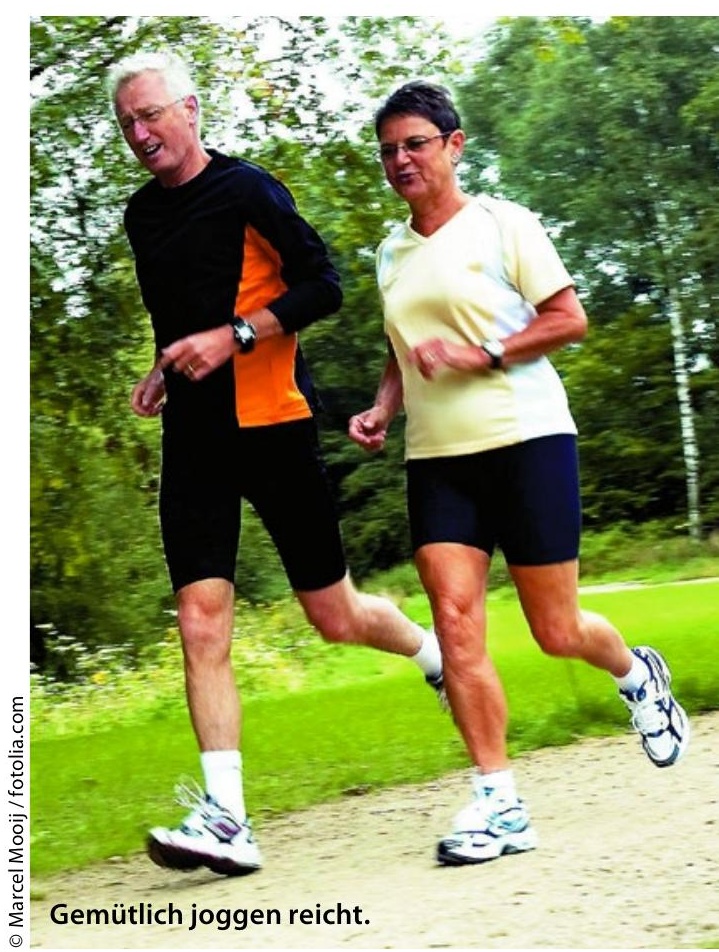

